regression model to investigate critical features associated with prognosis.

Results Patients with low MA (called intramuscular fat [IMF] deposition) had shorter median survival than nonIMF deposition (73 11.61 vs. 25226.56 days, $p=0.008$ ) as also observed shorter in patients with alpha-fetoprotein (AFP) level $\geq 200 \mathrm{ng} / \mathrm{ml}$ than AFP $<200 \mathrm{ng} / \mathrm{ml}$ (69 31.31 vs. 9286.03 days, $p=0.015)$ as depicted in IDDF2018ABS-0195 Figure 1. By univariate analysis, IMF deposition $(p=0.012), \quad H B V \quad(p=0.048)$, Child-Pugh $(C P)$ class B $(p=0.019), \quad$ CP class C $\quad(p<0.001)$, BCLC stage C $(p=0.009)$, BCLC stage $D(p<0.001)$ and AFP $\geq 200 \mathrm{ng} / \mathrm{ml}$ $(p=0.018)$ were significantly associated with mortality. However, multivariate analysis revealed that only IMF deposition (HR, 2.390; 95\% CI, 1.007-5.673; p=0.048), BCLC stage C (HR, 4.641; 95\% CI, 1.369-15.732; $\mathrm{p}=0.014)$ and BCLC stage D (HR, 14.681; 95\% CI, $3.158-68.253 ; \mathrm{p}=0.001)$ were independently associated with mortality.

Conclusions Muscle Attenuation and BCLC stage were significant independent predictors of survival in HCC Indonesian patients. External validation of these prognostic factors in larger cohorts of HCC patients is warranted.

\section{IDDF2018-ABS-0200 NON-LINEAR ASSOCIATION BETWEEN SERUM HEPATITIS B VIRUS DNA LEVELS AND HEPATOCELLULAR CARCINOMA RISK IN TREATMENT-NAIVE, NON-CIRRHOTIC CHRONIC HEPATITIS B PATIENTS}

${ }^{1} \mathrm{Gi}-\mathrm{Ae}$ Kim*${ }^{*}{ }^{2}$ Seungbong Han, ${ }^{3} \mathrm{G}$ wangHyeon Choi, ${ }^{3}$ Jonggi Choi, ${ }^{3}$ Young-Suk Lim. ${ }^{1}$ Health Screening and Promotion Center, Asan Medical Center, University of Ulsan College of Medicine, Korea, South; '2Department of Applied Statistics, Gachon University, Korea, South; ${ }^{3}$ Department of Gastroenterology, Liver Center, Asan Medical Center, University of Ulsan College of Medicine, Korea, South

\subsection{6/gutjn|-2018-IDDFabstracts.232}

Background The REVEAL-HBV studies on the natural history of chronic hepatitis $\mathrm{B}(\mathrm{CHB})$ showed a linear correlation between serum HBV DNA levels and the risk of hepatocellular carcinoma (HCC). However, virus titers above $10^{6}$ copies/ $\mathrm{mL}$ were not quantified, and most of the patients were HBeAg-negative (85\%). The progression of these HBeAg-negative patients would not be identical to that of young HBeAgpositive immune-tolerant phase patients.

Methods The study subjects were recruited from a historical cohort of 4367 treatment-naive, non-cirrhotic $\mathrm{CHB}$ patients (1240 HBeAg-positive and $3127 \mathrm{HBeAg}$-negative) with serum ALT levels lower than $2 \mathrm{x}$ upper limit of normal (females, $<19 \mathrm{IU} / \mathrm{mL}$; males, $<30 \mathrm{IU} / \mathrm{mL}$ ) at a tertiary referral hospital in Korea. Cox proportional hazards regression model predicted HCC risk considering sex, age, HBeAg status, HBV DNA levels, ALT levels, and platelet counts.

Results During the total follow-up of 23690 person-years, 221 patients (5.1\%) developed HCC. Old age, male gender, and lower platelet counts were found to be associated with an increased risk of HCC. Among HBeAg-negative patients, HBV DNA levels were linearly associated with increased risk of HCC. In contrast, among HBeAg-positive patients, HBV DNA levels were inversely associated with the risk of HCC. The risk of HCC was the lowest in $\mathrm{HBeAg}$ negative patients with HBV DNA level below $4 \log _{10} \mathrm{IU} / \mathrm{mL}$ (reference group) and was the highest in HBeAg positive patients with HBV DNA levels between $4 \log _{10} \mathrm{IU} / \mathrm{mL}$ and $6 \log _{10} \mathrm{IU} / \mathrm{mL}$ (HR 6.89; 95\% CI 4.20-11.29). HBeAg-positive patients with HBV DNA levels above $8 \log _{10} \mathrm{IU} / \mathrm{mL}$ showed the similar risk of HCC compared with the reference group (HR 1.36; 95\% CI $0.65-$ 2.84).

Conclusions In our cohort, the association between HBV DNA levels and HCC risk was not linear but was parabolic. HCC risk was the highest in patients with HBV DNA levels between $4 \log _{10} \mathrm{IU} / \mathrm{mL}$ and $6 \log _{10} \mathrm{IU} / \mathrm{mL}$. These data may help assess the HCC risk among the patients who are not subject to antiviral treatment which consequently calls attention to the necessity of developing a new treatment indication.

\section{IDDF2018-ABS-0201 FAECAL MICROBIOTA TRANSPLANTATION INDUCED HBSAG DECLINE IN HBEAG NEGATIVE CHRONIC HEPATITIS B PATIENTS AFTER LONG-TERM ANTIVIRAL THERAPY}

${ }^{1}$ Yurou Xie*, ${ }^{2}$ Zhangran Chen, ${ }^{1}$ Fei Zhou, 'Ligang Chen, ${ }^{2}$ Jianquan He, ${ }^{1}$ Chuanxing Xiao, ${ }^{1}$ Hongzhi Xu, ${ }^{1}$ Jianlin Ren, ${ }^{2}$ Xiang Zhang. 'Zhongshan Hospital Department of Gastroenterology, Xiamen University, Xiamen, China; ${ }^{2}$ Institute for Microbial Ecology, Medical College of Xiamen University, Xiamen, China

\subsection{6/gutjnl-2018-IDDFabstracts.233}

Background Serum HBsAg decline is an index for the effectiveness of antiviral treatment hepatitis B virus (HBV) e-antigen (HBeAg)-negative chronic hepatitis $\mathrm{B}(\mathrm{CHB})$ patients. Unfortunately, for entecavir (ETV), HBsAg decline or clearance only occurs in a minority of patients even after decades of antiviral therapy. Our previous study showed that Faecal microbiota transplantation (FMT) was able to induce $\mathrm{HBeAg}$ clearance in patients with positive $\mathrm{HBeAg}$ after long-term antiviral therapy. However, the effect of FMT on HBeAg negative $\mathrm{CHB}$ patients after long-term antiviral therapy is still unclear. Thus, we reported a case-controlled, open-label pilot trial of FMT for HBeAg negative CHB patients.

Methods We recruited 10 patients who remained HBsAg positive following $>1$ years of ongoing ETV antiviral therapy. 5 of them were enrolled in the FMT arm while other 5 were enrolled in the control arm. All patients went on their previous antiviral therapy. We performed FMT via nasojejunal tube for the FMT arm every 2 weeks. The faecal microbial community was analysed using Illumina Hisequencing of $16 \mathrm{~S}$ rDNA and bioinformatics methods. Serum HBsAg and endotoxin levels were monitored every 2 weeks.

Results Serum HBsAg declined gradually after each time of FMT in the FMT arm compared to control arm. FMT induced serum HBsAg decline up to $55.27 \% \pm 8.52 \%$ in the trial group accompanied with serum endotoxin decline while serum HBsAg in the control group increased 38.07\% which may closely be related to drug resistance. $16 \mathrm{~S} \mathrm{rDNA}$ analysis of the stools showed that gut microbiota in the FMT group transformed towards the donors. In particular, the proportion of Bifidobacterium, Streptococcus, Clostridium, Clostridiaceae, Blautia, Coprococcus and Megamonas in the gut microbiota of the FMT group decreased significantly towards the donors while the proportion of Bacteroides, Butyricimonas, Odoribacter, Prevotella, Parabacteroides, Anaerostipes, Oscillospira, Ruminococcaceae and Sutterella increased towards the donors. Conclusions This study suggested that HBsAg decline in the serum of $\mathrm{HBeAg}$ negative $\mathrm{CHB}$ patients were detected after several times of FMT accompanied with serum endotoxin 


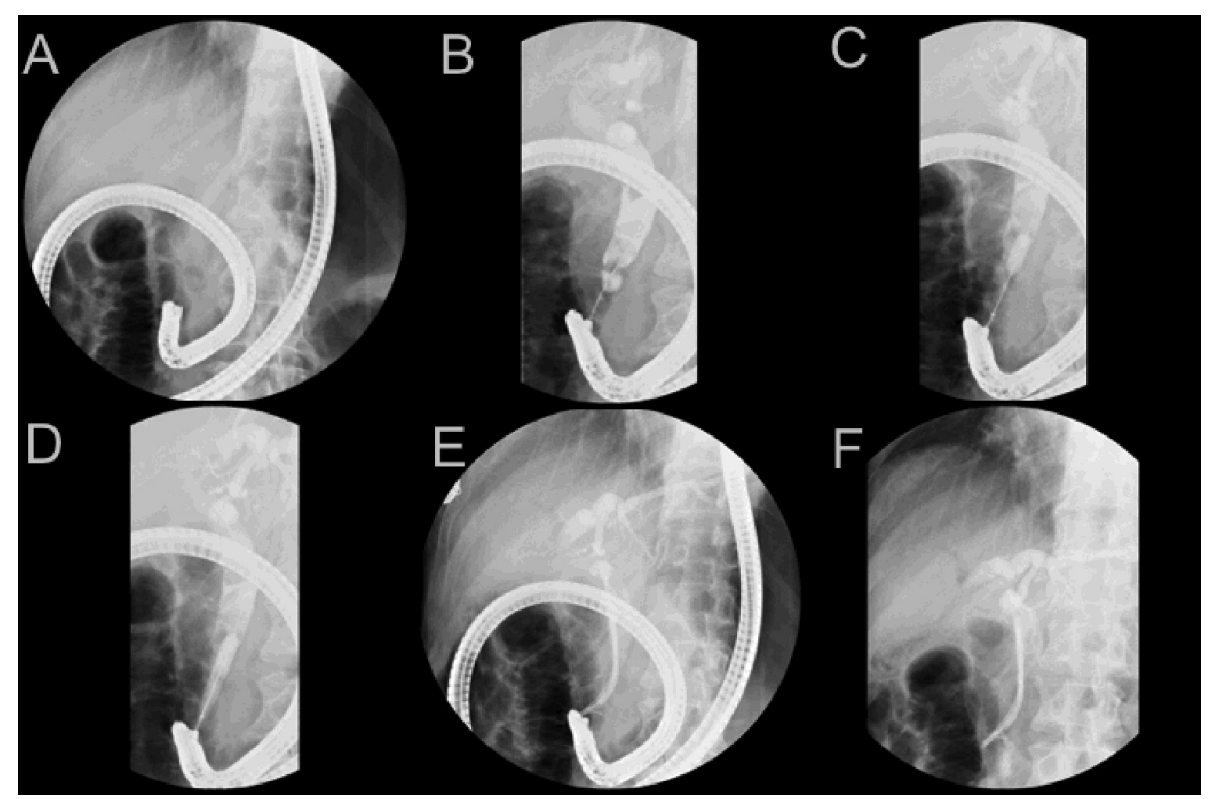

\section{Abstract IDDF2018-ABS-0202 Figure 1}

decline and the gut microbiota prone to the donors. Gut microbiota may be a new target for the treatment of $\mathrm{HBeAg}$ negative $\mathrm{CHB}$.

\section{IDDF2018-ABS-0202 OUTCOMES OF THE CONVENTIONAL SIDE- VIEWING DUODENOSCOPE FOR ERCP IN BILLROTH II GASTRECTOMY PATIENTS}

Like Bie*. Digestive Endoscopy Center, Department of Gastroenterology, Ruijin Hospital, Affiliated to Shanghai Jiao Tong University School of Medicine, China

\subsection{6/gutjnl-2018-IDDFabstracts.234}

Background Endoscopic retrograde cholangiopancreatography (ERCP) in patients with prior Billroth II gastrectomy is a challenging procedure for the endoscopist. The aim of this study was to evaluate the usefulness of the conventional side-viewing duodenoscope for ERCP in Billroth II gastrectomy patients.

Methods The records of patients with previous Billroth II gastrectomy referred for ERCP between July 2008, and January 2017 were retrospectively reviewed. The outcomes combined with risk factors for ERCP-related adverse events were analysed.

Results A total of 106 consecutive patients undergoing 123 ERCP procedures using a conventional side-viewing duodenoscope were enrolled. The success rate of the selective entrance to the afferent loop and access to the papilla was 93\%, 89\%, respectively. In cases of successful access, the rate of successful selective cannulation was $96.6 \%$. The rate of clinical success was $84 \%$. Adverse events related to ERCP occurred in 20 procedures (16.2\%): pancreatitis, asymptomatic hyperamylasemia, perforation and cholangitis were in 6 procedures $(4.9 \%)$, 11 procedures (8.9\%), 1 procedures $(0.8 \%), 2$ procedures (1.6\%), respectively. Female (odds ratio [OR], 4.210; 95\% confidence interval $[\mathrm{CI}], 1.581-11.214 ; \mathrm{p}=0.004)$ and periampullary diverticulum (OR, 4.373; 95\% CI, 1.713-11.164; $\mathrm{p}=0.002$ ) were significantly associated with the incidence of ERCP-related adverse events.

Conclusions The conventional side-viewing duodenoscope can be safely and effectively used to perform ERCP in most Billroth II gastrectomy patients. Female and periampullary diverticulum might be significant risk factors for ERCP-related adverse events.

\section{IDDF2018-ABS-0219 ASSESSMENT OF CORRELATION BETWEEN NON-ALCOHOLIC FATTY LIVER DISEASE AND SEVERITY OF CORONARY ARTERY DISEASE IN YOUNG ACUTE CORONARY SYNDROME PATIENTS}

${ }^{1}$ Alyaa Hassan Ali*, ${ }^{2}$ Shawal Mohamed Faizal, ${ }^{1}$ Khairul Najmi Muhammad Nawawi, ${ }^{1}$ Wong Zhiqin, ${ }^{1}$ Ngiu Chai Soon, ${ }^{3}$ Nur Yazmin Yaacob, ${ }^{4}$ Shamsul Azhar Shah, ${ }^{2}$ Hamad Hamdi Che Hassan, ${ }^{2}$ Oteh Maskon, 'Raja Affendi Raja Ali. ' Gastroenterology Unit, Faculty of Medicine, Universiti Kebangsaan Malaysia Medical Centre, Kuala Lumpur, Malaysia; ${ }^{2}$ Cardiology Unit, Faculty of Medicine, Universiti Kebangsaan Malaysia Medical Centre, Kuala Lumpur, Malaysia; ${ }^{3}$ Department of Radiology, Faculty of Medicine, Universiti Kebangsaan Malaysia Medical Centre, Kuala Lumpur, Malaysia; ${ }^{4}$ Department of Community Health, Faculty of Medicine, Universiti Kebangsaan Malaysia Medical Centre, Kuala Lumpur, Malaysia

\subsection{6/gutjnl-2018-IDDFabstracts.235}

Background Coronary artery disease (CAD), which clinically manifested as an acute coronary syndrome (ACS) is an important extrahepatic cardiovascular complication of non-alcoholic fatty liver disease (NAFLD). To date, little is known about the correlation between young patients with ACS and NAFLD. Aim to assess the correlation between NAFLD and severity of CAD in young ACS patients and determine the prevalence of NAFLD in this cohort.

Methods This cross-sectional study included young (45 years and less) ACS patients. Coronary angiogram was performed to assess CAD severity, and complexity determined by SYNTAX score. CAD severity was classified into: no apparent CAD, mild CAD, single vessel disease (SVD) and multi-vessel disease 